\title{
Relation of familial patterns of coronary heart disease, stroke, and diabetes to subclinical atherosclerosis: the multi-ethnic study of atherosclerosis
}

Maren T. Scheuner, MD, MPH ${ }^{1}$, Claude Messan Setodji, PhD ${ }^{1}$, James S. Pankow, PhD, MPH , Roger S. Blumenthal, $\mathrm{MD}^{3}$, and Emmett Keeler, $P h D^{1}$

\begin{abstract}
Purpose: To investigate the possibility that family history beyond early-onset coronary heart disease might contribute to coronary heart disease susceptibility, we studied associations between additional family history and the coronary artery calcium score. Methods: Associations between coronary artery calcium score and self-reports of coronary heart disease, stroke, and diabetes in first-degree relatives of 5264 nondiabetic subjects were assessed using logistic and linear regression adjusting for risk factors; adjusted mean coronary artery calcium score estimates were determined by pooling results. Results: Family history of coronary heart disease alone and in combination with diabetes and/or stroke was significantly associated with a positive coronary artery calcium score compared with no family history with odds ratios ranging from 1.7 (95\% Cl: 1.3-2.3) to 1.9 (95\% Cl: 1.6-2.3) and adjusted mean coronary artery calcium score estimates ranging from 137 (95\% Cl: 101-173) to 184 (95\% Cl: 143-226). Associations between family history of coronary heart disease and coronary artery calcium score were significant regardless of age at onset, sex, lineage, or number of relatives with coronary heart disease. The association between family history of diabetes only and coronary artery calcium score was also significant (OR, 1.3; 95\% Cl: 1.1-1.7) with an adjusted mean coronary artery calcium score estimate of 122 (95\% Cl: 93-151). Generally, family history of stroke had nonsignificant associations with coronary artery calcium score. Conclusions: Numerous family history variables in addition to early-onset coronary heart disease are associated with subclinical atherosclerosis. Our results have implications for improving coronary heart disease risk assessment. Genet Med
\end{abstract} 2008:10(12):879-887.

Key Words: family history, coronary heart disease, stroke, diabetes, subclinical atherosclerosis

Family history is a well-known and significant risk factor for coronary heart disease (CHD). However, many studies that have investigated family history as a risk factor have limited the definition of a positive family history to premature CHD in first-degree relatives (defined as CHD occurring before age 55 years in men and before age 65 years in women). Few studies have considered other definitions of a positive family history for the outcome of $\mathrm{CHD},{ }^{1}$ and none have assessed a broader definition of a positive family history for the outcome of sub-

\footnotetext{
From the ${ }^{1}$ RAND Corporation, Santa Monica, California; ${ }^{2}$ Division of Epidemiology and Community Health, University of Minnesota, Minneapolis, Minnesota; and ${ }^{3}$ Department of Cardiology, Johns Hopkins University, Baltimore, Maryland.

Maren T. Scheuner, MD, MPH, RAND Corporation, 1776 Main Street, PO Box 2138, Santa Monica, CA 90407-2138. E-mail: scheuner@rand.org.

Disclosure: The authors declare no conflict of interest.

Submitted for publication June 12, 2008.

Accepted for publication September 18, 2008.

DOI: 10.1097/GIM.0b013e31818e639b
}

clinical atherosclerosis, which is increasingly becoming the target for risk factor modification..$^{2-4}$

Understanding how risk factors contribute to cardiovascular disease risk is important to risk assessment and tailoring recommendations for risk factor modification. However, widely used risk stratification guidelines for the primary prevention of $\mathrm{CHD}$ lack family history altogether or they include a limited definition of positive family history. For example, the Framingham risk score, which predicts the 10 -year risk of a clinical CHD event such as myocardial infarction or sudden death, is based on the traditional risk factors of age, gender, total or low-density lipoprotein (LDL) cholesterol, high-density lipoprotein (HDL) cholesterol, blood pressure, and smoking-family history is not included. ${ }^{5}$ The National Cholesterol Education Program Adult Treatment Panel III guidelines ${ }^{6}$ only consider family history of early-onset CHD (age of onset $<55$ years for men and $<65$ years for women) in a first-degree relative as a risk factor. $\mathrm{CHD}$ at later ages of onset is not included, nor is a family history of related conditions such as stroke and diabetes. 
To investigate the possibility that family history beyond earlyonset CHD in first-degree relatives might contribute to subclinical atherosclerosis, we studied the associations between coronary artery calcium scores (CACS) and self-reports of CHD, stroke, and diabetes in first-degree relatives among nondiabetic subjects participating in the Multi-Ethnic Study of Atherosclerosis (MESA). Positive associations could provide the rationale for evaluating the added value of family history variables within existing cardiovascular risk stratification models.

\section{MATERIALS AND METHODS}

This study is an ancillary study to MESA, a cohort study that enrolled 6814 subjects without clinically apparent atherosclerotic vascular disease (e.g., no history of heart attack, angina, stroke, transient ischemic attack, or heart failure), age 45-84, from six sites across the United States (Columbia University, Johns Hopkins University, Northwestern University, University of Minnesota, UCLA, and Wake Forest University); 53\% of enrolled subjects were women, and $62 \%$ were ethnic/racial minorities. The purpose of MESA is to study the relationship between cardiovascular risk factors and subclinical atherosclerosis measured periodically using electron beam computed tomography (EBCT) or multidetector computed tomography (MDCT) and other modalities such as ultrasound and magnetic resonance imaging over a 10-year period (1999-2009) with four clinical examinations scheduled 18-24 months apart.

This study utilized data collected in exams 1 and 2 from nondiabetic MESA subjects to assess the relationships between family history and baseline measures of CACS using regression analyses adjusting for demographic and personal risk factors. MESA subjects were excluded if they had previously diagnosed diabetes, a fasting glucose $\geq 126 \mathrm{mg} / \mathrm{dL}$, or used a hypoglycemic medication $(n=995)$ and if information regarding family history $(n=483)$ or covariates included in the regression models $(n=72)$ was missing. This study has been reviewed and approved by the RAND Human Subjects Protection Committee.

\section{Demographic factors}

Information about age, gender, ethnicity, marital status, educational level, income level, and medical history was obtained by questionnaires in exam 1 . Laboratory test results were also measured in exam 1 and included fasting lipid levels (total cholesterol, LDL cholesterol, HDL cholesterol, and triglycerides) and glucose. Current smoking was defined as having smoked a cigarette in the last 30 days. Resting blood pressure was measured three times in the seated position, and the average of the second and third readings was recorded. Hypertension was defined as a systolic blood pressure $\geq 140 \mathrm{mmHg}$, diastolic blood pressure $\geq 90 \mathrm{mmHg}$, or use of medication prescribed for hypertension. Hypercholesterolemia was defined as those participants taking medication to lower cholesterol. Use of medications was based on clinical staff entry of prescribed medications verified by the staff. Body mass index was calculated as weight $(\mathrm{kg}) /$ height $^{2}\left(\mathrm{~m}^{2}\right)$.

\section{Family history}

Detailed information on family histories of CHD, stroke, and diabetes was ascertained in exam 2 using a structured survey instrument. Subjects were asked if their mother, father, siblings, or children have had CHD defined as a heart attack, myocardial infarction, or cardiac procedures (coronary bypass surgery, balloon angioplasty, intracoronary stenting); stroke, cerebral hemorrhage, or brain attack; or diabetes or high blood sugar. Response options were yes, no, and don't know. If a subject reported a disease in a relative, the age at diagnosis was also ascertained. Reports of CHD and stroke occurring in relatives before age 25 were excluded in our family history assessment, as we suspected these were most likely cases of congenital heart disease, congenital cerebrovascular malformations, or single gene forms of cardiovascular disease. Relatives with a first occurrence of $\mathrm{CHD}$ or stroke after age 85 were not included. For diabetes, we excluded diagnoses occurring in relatives before age 20 as we considered this history more consistent with type 1 diabetes than type 2 diabetes. The latter was of greater interest because it is closely linked to atherosclerosis through insulin resistance.

Eight different family history variables were developed that considered the presence or absence of CHD, diabetes, and/or stroke at any age of onset in first-degree relatives including (1) no family history of CHD, diabetes, or stroke; (2) family history of CHD only; (3) family history of diabetes only; (4) family history of stroke only; (5) family history of CHD and diabetes; (6) family history of CHD and stroke; (7) family history of stroke and diabetes; and (8) family history of CHD, stroke, and diabetes.

We also conducted analyses that assessed the association between CACS and the number $(0,1,2,3$, or more), type (parent, siblings, children), lineage (maternal, paternal, nuclear), and youngest age of onset of relatives with CHD, stroke, or diabetes compared to having no affected relative. Maternal lineage included families with an affected mother with or without affected siblings or children. Paternal lineage included families with an affected father with or without affected siblings or children. Nuclear lineage included families with affected siblings or children and no affected parent. To investigate the influence of age of disease onset thresholds in relatives, we used the nonparametric technique of generalized additive models ${ }^{7}$ to $\mathrm{ex}^{-}$ plore the functional form of the relationship between age of disease onset and a positive CACS. This relationship is estimated using smoothing operations, and graphic displays were used to select the different age of onset thresholds. For all three conditions, three age of onset thresholds were identified as age $<40$ years, $40-60$ years, and 60 years and older. We observed increasing, flat, and then decreasing linear associations across these age groups. We did not observe differences when we assessed the age of onset thresholds for each condition according to the sex of the relative or the sex of the MESA participant. 


\section{Coronary artery calcium score}

The mean Agatston, phantom-adjusted CACS was the outcome measure used in the analyses of this study. Computed tomography scanning of the chest was performed on the MESA cohort either with an ECG-triggered (at $80 \%$ of the RR interval) EBCT scanner (Chicago, Los Angeles, and New York field centers; Imatron C-150, Imatron) or with prospectively ECGtriggered scan acquisition at $50 \%$ of the RR interval with a MDCT system that acquired four simultaneous 2.5-mm slices for each cardiac cycle in a sequential or axial scan mode (Baltimore, Forsyth County, and St. Paul field centers; Lightspeed, General Electric or Siemens, Volume Zoom). Each participant was scanned twice. Scans were read centrally at the Los Angeles Biomedical Research Institute at Harbor-UCLA Medical Center to identify and quantify coronary calcification. The CACS measurements among scanning centers and between participants were adjusted with a standard calcium phantom scanned simultaneously with each participant. The mean Agatston score was used in all analyses. Agreement with regard to presence of CACS was high (kappa statistic $0.90-0.93$ between and within readers), and the intraclass correlation coefficient for the Agatston score between readers was 0.99. Concordance for presence of coronary artery calcification between duplicate scans was high and similar for both EBCT and MDCT (96\%, kappa $=0.92) .^{8}$

\section{Statistical analyses}

SAS software (version 9.1) was used for all statistical analyses. Basic descriptive statistics were performed to describe the distribution, mean value, and range of the demographic factors, personal risk factors, family histories, and CACS. Chisquare and $t$ tests were used to test for any difference in the outcomes between different ethnic/racial groups and any other group stratification. When appropriate, the significance of the results presented in the text and tables has been adjusted for multiple comparisons. The Bonferroni method was applied to adjust the threshold of statistical significance when multiple comparisons were made.

The association between CACS and family history was assessed using a two-part model structure. ${ }^{9-11}$ Because many participants had a CACS of zero, we first assessed the association between having a positive CACS and family history using logistic regression, and then for those subjects with a positive CACS, we modeled their continuous score using a linear regression model. The positive scores were skewed, therefore a log transformation of the outcome was used to help stabilize the error variance and improve the fit with the linearity and normality assumptions. To appreciate the magnitude of the different estimates derived from both the logistic and the linear regression models, we report predictive margins or adjusted mean estimates for the CACS. ${ }^{12}$ These adjusted mean estimates represent average predicted outcomes if every participant in the sample would have the risk factor of interest (e.g., family history of CHD only), and these estimates are equivalents of the model coefficient estimates in the outcome scale. So, for the first part of the two-part model (logistic), adjusted proportions or probabilities equivalent to the estimated odds ratios are used, and for the second part of the two-pat model (linear), predictions were back-transformed into the CACS scale (where the score was $>0$ ) using the smearing estimate technique $^{13}$ and again presented as adjusted means. The combined associations between family history and CACS using both the zero and the nonzero scores were also estimated considering that at a participant prediction level the combined estimated score can be obtained as the product of the probability of having a nonzero score and the estimate of the participant's score using the two-part model: $E(\mathrm{CACS} \mid$ Covariates $)=\operatorname{Prob}(\mathrm{CACS}>0) \times$ $E$ (CACS $\mid$ Covariates, CACS $>0$ ).

In all of the models, we adjusted for age, sex, education, income, marital status, ethnicity/race, study site, family size, current smoking, LDL cholesterol, HDL cholesterol, triglycerides, systolic and diastolic blood pressure, and body mass index. Models adjusted for sociodemographic factors only, excluding cardiovascular risk factors, produced similar results to the fully adjusted models, and are therefore not presented. Interactions between family history of CHD, stroke and/or diabetes and age, sex and ethnicity/race on the outcome of CACS were evaluated by the addition of interaction terms into regression models.

\section{RESULTS}

Table 1 provides a description of the 5264 nondiabetic MESA subjects eligible for this study. The mean age was 61.6 years (range, $44-84$ ), 47\% were men, and 57\% were nonwhite. The majority (67\%) had more than a high school education, and a range of annual incomes was represented. The mean number of first-degree family members per subject was 7.8 (range 1-35). About 37\% of the subjects were current cigarette smokers. Personal history of hypertension or use of blood pressure-lowering medication was reported by $41 \%$, and use of cholesterol-lowering medication was reported by $14.4 \%$. Slightly more than half (53\%) of subjects had a CACS of 0 . The mean CACS was 124 (SD, 371; median, 0; range, 0-6316), and the mean value for subjects with a positive CACS (i.e., CACS $>0$ ) was 264 (SD, 505; median, 79; range, 1-6316). Subjects with a positive CACS were more likely to be older, men, white, current smokers, and to have lower annual incomes and personal history of hypertension and lipid disorders.

Tests for goodness of fit for both the logistic and the linear regression models comparing likelihood ratios and $R^{2}$ values found that models including family history defined as having at least one first-degree relative with $\mathrm{CHD}$, stroke, or diabetes were significantly better than models that did not include family history $(P<0.0001$ and $P<0.001$, respectively). The odds ratios and CACS estimates describing the associations of family history of $\mathrm{CHD}$, stroke, and/or diabetes in at least one firstdegree relative at any age of onset compared with no family history and adjusted for demographic factors, personal risk factors, and multiple comparisons are shown in Table 2. Family history of CHD alone and in combination with diabetes 
Table 1

Characteristics of nondiabetic MESA subjects at baseline

\begin{tabular}{|c|c|c|c|}
\hline Characteristics & $\begin{array}{l}\mathrm{CAC}=0 \\
(N=2780)\end{array}$ & $\begin{array}{l}\text { CAC }>0 \\
(N=2484)\end{array}$ & $\begin{array}{c}\text { Total } \\
(N=5264)\end{array}$ \\
\hline Age: mean (SD), range $(\mathrm{yr})^{a}$ & $57.6(9), 44-84$ & $66.1(9.7), 44-84$ & $61.6(10.2), 44-84$ \\
\hline Male $(\%)^{a}$ & 36.5 & 58.2 & 46.7 \\
\hline \multicolumn{4}{|l|}{ Ethnicity/race $(\%)^{a}$} \\
\hline African-American & 29.1 & 21.4 & 25.5 \\
\hline Asian-American & 11.7 & 11.4 & 11.6 \\
\hline White & 36.7 & 49.4 & 42.7 \\
\hline Hispanic & 22.4 & 17.9 & 20.3 \\
\hline \multicolumn{4}{|l|}{ Education $(\%)^{b}$} \\
\hline Less than high school & 15.1 & 14.6 & 14.9 \\
\hline High school graduate & 16.2 & 19.6 & 17.8 \\
\hline More than high school & 68.7 & 65.8 & 67.3 \\
\hline \multicolumn{4}{|l|}{ Marital status (\%) } \\
\hline Married or living as married & 61.8 & 61.8 & 61.8 \\
\hline Divorced, widowed or separated & 28.7 & 29.3 & 29.0 \\
\hline Never married & 8.5 & 8.3 & 8.4 \\
\hline \multicolumn{4}{|l|}{ Income $(\%)^{a}$} \\
\hline Less than $\$ 25,000$ & 24.5 & 30.2 & 27.2 \\
\hline$\$ 25,000-\$ 49,999$ & 28.3 & 27.7 & 28.1 \\
\hline$\$ 50,000-\$ 74,999$ & 18.5 & 15.9 & 17.3 \\
\hline$\$ 75,000-\$ 99,999$ & 10.4 & 9.1 & 9.8 \\
\hline More than $\$ 100,000$ & 15.5 & 13.7 & 14.7 \\
\hline Number of relatives: mean (SD), range ${ }^{c}$ & $7.9(3.6), 1-35$ & $7.7(3.5), 1-21$ & $7.8(3.5), 1-35$ \\
\hline \multicolumn{4}{|l|}{ Smoking status $(\%)^{a}$} \\
\hline Current & 30.9 & 43.5 & 36.9 \\
\hline Former & 12.9 & 12.4 & 12.6 \\
\hline Never & 56.2 & 44.1 & 50.4 \\
\hline Personal history of hypertension $(\%)^{a, d}$ & 31.5 & 50.8 & \\
\hline $\begin{array}{l}\text { Systolic blood pressure: mean (SD), range } \\
\qquad(\mathrm{mm} \mathrm{Hg})^{a}\end{array}$ & $121(20), 78-231$ & $129(21), 75-218$ & $125(21), 75-231$ \\
\hline $\begin{array}{l}\text { Diastolic blood pressure: mean (SD), range } \\
\qquad(\mathrm{mm} \mathrm{Hg})^{a}\end{array}$ & $71(10), 41-106$ & $73(10), 41-110$ & $72(10), 41-110$ \\
\hline Personal history of lipid disorder $(\%)^{a, e}$ & 9.8 & 19.7 & 14.5 \\
\hline LDL cholesterol: mean $(\mathrm{SD})$, range $(\mathrm{mg} / \mathrm{dL})^{a}$ & 117 (30), 21-252 & $119(31), 20-284$ & $118(31), 20-284$ \\
\hline HDL cholesterol: mean $(\mathrm{SD})$, range $(\mathrm{mg} / \mathrm{dL})^{a}$ & 53 (15), 21-142 & $50(15), 15-127$ & $52(15), 15-142$ \\
\hline Triglycerides: mean $(\mathrm{SD})$, range $(\mathrm{mg} / \mathrm{dL})^{a}$ & $120(63), 21-400$ & $126(64), 23-391$ & $123(64), 21-400$ \\
\hline BMI: mean $(\mathrm{SD})$, range $\left(\mathrm{kg} / \mathrm{m}^{2}\right)$ & $28(6), 15-54$ & $28(5), 16-55$ & $28(5), 15-55$ \\
\hline $\begin{array}{l}\text { Coronary calcium score: mean (SD), } \\
\text { median, range }{ }^{a}\end{array}$ & 0 & $264(505), 79,1-6316$ & $124(371), 0-6316$ \\
\hline
\end{tabular}

${ }^{a}$ Comparison of CAC $=0$ to CAC $>0$ group, $P<0.001$.

${ }^{b}$ Comparison of $\mathrm{CAC}=0$ to $\mathrm{CAC}>0$ group, $P<0.01$.

${ }^{c}$ Comparison of $\mathrm{CAC}=0$ to CAC $>0$ group, $P<0.05$.

${ }^{d}$ Includes use of blood pressure-lowering medication.

${ }^{e}$ Includes use of cholesterol-lowering medication.

MESA, multi-ethnic study of atherosclerosis; SD, standard deviation; LDL, low-density lipoprotein; HDL, high-density lipoprotein; BMI, body mass index. 
Table 2

Associations between CACS and family history of CHD, stroke, and diabetes in MESA subjects at baseline

\begin{tabular}{|c|c|c|c|}
\hline Family history of: & $\begin{array}{l}\text { Odds ratio }(95 \% \mathrm{CI})^{a} \text { CACS }>0 \text { vs. } \\
\qquad \text { CACS }=0\end{array}$ & $\begin{array}{c}\text { CACS estimate }(95 \% \mathrm{CI}) \text { when } \\
\text { CACS }>0^{b}\end{array}$ & $\begin{array}{c}\text { Adjusted mean CACS } \\
\text { estimate }^{c}\end{array}$ \\
\hline No CHD, diabetes, or stroke $(n=1,688)$ & 1.00 & $241(206-275)$ & $105(91-119)$ \\
\hline CHD only $(n=966)$ & $1.9(1.6-2.3)^{d}$ & $332(275-389)^{d}$ & $169(143-195)$ \\
\hline Diabetes only $(n=523)$ & $1.3(1.1-1.7)^{e}$ & $259(197-321)$ & $122(93-151)$ \\
\hline Stroke only $(n=515)$ & $1.1(0.9-1.4)$ & $225(174-275)$ & $101(79-124)$ \\
\hline CHD and diabetes $(n=477)$ & $1.8(1.4-2.2)^{d}$ & $367(280-454)^{d}$ & $184(143-226)$ \\
\hline CHD and stroke $(n=502)$ & $1.8(1.4-2.3)^{d}$ & $327(254-400)^{f}$ & $164(130-199)$ \\
\hline Stroke and diabetes $(n=236)$ & $1.1(0.8-1.5)$ & $221(143-298)$ & $99(64-134)$ \\
\hline CHD, diabetes, and stroke $(n=357)$ & $1.7(1.3-2.3)^{d}$ & $273(199-347)$ & $137(101-173)$ \\
\hline
\end{tabular}

For each analysis, all $P$ values were adjusted for two comparisons (i.e., the two models used-logistic regression and linear regression) using the Bonferroni method with a significance threshold value of 0.025 .

${ }^{a}$ Part 1 of the two-part model: odds ratios for a positive CACS derived from logistic regression adjusting for age, sex, ethnicity/race, education, income, marital status, study site, family size, current smoking status, LDL cholesterol, HDL cholesterol, triglycerides, systolic blood pressure, diastolic blood pressure, and body mass index. ${ }^{b}$ Part 2 of the two-part model: CACS estimates derived from linear regression adjusting for age, sex, ethnicity/race, education, income, marital status, study site, family size, current smoking status, LDL cholesterol, HDL cholesterol, triglycerides, systolic blood pressure, diastolic blood pressure, and body mass index.

${ }^{c}$ Adjusted mean CACS estimate is the mean value calculated by pooling the results from the logistic and the log-transformed linear regression models. All $P$ values were adjusted for multiple comparisons (i.e., the two models used-logistic regression and linear regression).

${ }^{d} P<0.01$, statistically significant after adjustment for two comparisons using the Bonferroni method.

${ }^{e} P=0.02$, statistically significant after adjustment for two comparisons using the Bonferroni method.

${ }^{f} P=0.01$, statistically significant after adjustment for two comparisons using the Bonferroni method.

CACS, coronary artery calcification score; CHD, coronary heart disease; MESA, multi-ethnic study of atherosclerosis; CI, confidence interval.

and/or stroke was significantly associated with a positive CACS with odds ratios ranging from 1.7 (95\% CI: $1.3-2.3)$ to 1.9 (95\% CI: 1.6-2.3) and adjusted mean CACS estimates ranging from 137 (95\% CI: 101-173) to 184 (95\% CI: 143-226). A significant association of lesser magnitude was also observed between family history of diabetes only and a positive CACS (OR, 1.3; 95\% CI: 1.1-1.7) with an adjusted mean CACS estimate of 122 (95\% CI: 93-151) for these participants. However, the addition of family history of diabetes to family history of CHD did not substantially change the odds ratios or CACS estimates observed given family history of $\mathrm{CHD}$ alone. Family history of stroke only was not significantly associated with a positive CACS, and adjusted mean CACS estimates observed with family history of CHD and/or diabetes were if anything diminished by including family history of stroke. Because $\mathrm{CHD}$ has different prevalence rates according to age, sex, and ethnicity, we evaluated potential interactions between these demographic variables and the different family history combinations of CHD, stroke, and/or diabetes for the outcome of a positive CACS. We found no significant interactions and for this reason we did not present the results stratified by these variables, because any heterogeneity of associations by ethnicity/race, sex, or age could be due to chance.

To examine whether specific CHD, stroke, and diabetes family history variables - such as the youngest age of onset, number, sex, type, or lineage of affected relatives- had significant associations with CACS, we assessed the odds ratios for a positive CACS and calculated the mean CACS given these different family history variables compared to having no family history of each condition adjusting for demographics, personal risk factors, and multiple comparisons.
All of the associations between CACS and the specific CHD family history variables relating to youngest age at onset, number of affected relatives, and sex of affected relatives were significantly associated with a positive CACS (Table 3 ). The magnitude of the adjusted mean CACS estimates and associations with a positive CACS increased as the age of onset decreased and the number of relatives with CHD increased. Adjusted mean CACS estimates were $>200$ when there were both parents and siblings affected, both maternal and paternal relatives affected, three or more affected relatives, and when the youngest age of CHD onset was $<40$. This was about twice the CACS estimated in the absence of a family history of CHD. For the family history variables describing the type of affected relatives and lineage of affected relatives, all were significantly associated with a positive CACS; however, when only siblings were affected with CHD and when both maternal and paternal relatives were affected, the significance was at the threshold level when correcting for multiple comparisons.

There were no significant associations between a positive CACS and the specific stroke family history variables relating to age at onset, and number, sex, type, and lineage of affected relatives. The adjusted mean CACS estimates given a family history of stroke were generally similar to or less than the CACS estimate of about 133 for subjects without a family history of stroke. The stroke family history variable having the greatest adjusted mean CACS estimate of 151 (95\% CI: 114-188) was observed when the youngest age at stroke onset was between 40 and 60 years.

In considering the different diabetes family history variables, only one was significantly associated with a positive CACS, and that was having male relatives with diabetes, which 
Table 3

Odds ratios and CACS estimates according to number, sex, type, lineage, and age at onset of relatives with CHD, stroke, or diabetes in MESA subjects at baseline

\begin{tabular}{|c|c|c|c|}
\hline Family history variables & $N$ & $\begin{array}{c}\text { Odds ratio } \\
\text { CAC }>0(95 \% \text { CI })\end{array}$ & $\begin{array}{c}\text { Mean adjusted CACS } \\
\text { estimate }^{b}(95 \% \text { CI })\end{array}$ \\
\hline \multicolumn{4}{|l|}{ Youngest age at onset } \\
\hline No affected relative & 2962 & 1.0 & $106(94-117)$ \\
\hline Age $<40$ & 108 & $2.7(1.7-4.2)^{g}$ & $220(125-315)$ \\
\hline Age $40-60$ & 881 & $1.7(1.4-2.0)^{g}$ & $173(144-203)$ \\
\hline Age $>60$ & 1287 & $1.6(1.4-1.9)^{g}$ & $160(138-182)$ \\
\hline \multicolumn{4}{|l|}{ Number of affected relatives } \\
\hline No affected relative & 2962 & 1.0 & $105(94-117)$ \\
\hline 1 & 1589 & $1.6(1.4-1.8)^{g}$ & $148(128-167)$ \\
\hline 2 & 506 & $1.8(1.5-2.3)^{g}$ & $193(152-233)$ \\
\hline $3+$ & 207 & $2.4(1.7-3.4)^{g}$ & $244(172-316)$ \\
\hline \multicolumn{4}{|l|}{ Sex of affected relative } \\
\hline No affected relative & 2962 & 1.0 & $106(95-118)$ \\
\hline Male & 1241 & $1.5(1.3-1.8)^{g}$ & $154(131-176)$ \\
\hline Female & 603 & $1.8(1.5-2.2)^{g}$ & $155(125-185)$ \\
\hline Male and Female & 458 & $2.0(1.5-2.5)^{g}$ & $222(173-270)$ \\
\hline \multicolumn{4}{|l|}{ Type of affected relative } \\
\hline No affected relative & 2,962 & 1.0 & $106(94-117)$ \\
\hline Parents and no siblings ${ }^{c}$ & 1,306 & $1.6(1.4-1.9)^{g}$ & $156(133-180)$ \\
\hline Siblings and no parents ${ }^{c, d}$ & 580 & $1.6(1.3-2.0)^{h}$ & $158(128-187)$ \\
\hline Parents and siblings ${ }^{c}$ & 416 & $2.1(1.6-2.7)^{g}$ & $206(160-251)$ \\
\hline \multicolumn{4}{|l|}{ Lineage of affected relative } \\
\hline No affected relative & 2,962 & 1.0 & $106(95-118)$ \\
\hline Maternal $^{e}$ & 560 & $1.8(1.4-2.2)^{g}$ & $169(134-204)$ \\
\hline Paternal $^{e}$ & 956 & $1.7(1.4-2.0)^{g}$ & $155(130-181)$ \\
\hline Maternal and paternal ${ }^{e}$ & 206 & $1.6(1.2-2.3)^{h}$ & $264(174-353)$ \\
\hline Nuclear $^{f}$ & 580 & $1.6(1.3-2.0)^{g}$ & $157(127-187)$ \\
\hline
\end{tabular}

For each analysis, all $P$ values were adjusted for five comparisons (i.e., the models considering the five different family history variables of youngest age at onset, number of relatives affected, sex of affected relatives, type of affected relatives, and lineage of affected relatives) using the Bonferroni method with a significance threshold value of 0.01 .

${ }^{a}$ Adjusted for age, sex, education, income, marital status, study site, family size, current smoking status, LDL cholesterol, HDL cholesterol, triglycerides, systolic blood pressure, diastolic blood pressure, and body mass index. ${ }^{b}$ Mean CACS estimate is the mean value calculated by pooling the results from the logistic and the log-transformed linear models.

${ }^{c}$ Can include children.

${ }^{d}$ Includes 48 subjects with only children affected with CHD.

${ }^{e}$ Can include siblings and children with CHD.

Includes siblings and/or children with CHD but no affected parents.

${ }^{g} P<0.01$, statistically significant after adjustment for five multiple comparisons using the Bonferroni method.

${ }^{h} P=0.01$, at the threshold of statistical significance after adjustment for five comparisons using the Bonferroni method.

CACS, coronary artery calcification score; CHD, coronary heart disease; CI, confidence interval.

was associated with a 1.4-fold (95\% CI: 1.1-1.7) increase for a positive CACS. The greatest adjusted mean CACS estimate of 157 (95\% CI: 125-188) was observed when male relatives were affected compared with a CACS estimate of about 129 when there was no family history of diabetes. We observed a similar trend when fathers were affected with diabetes $(\mathrm{OR}=1.4 ; 95 \%$ CI: 1.0-1.7); however, the association did not reach significance after adjustment for multiple comparisons, and the ad- 
justed mean CACS estimate was not substantially increased (132; 95\% CI: 95-169). There was no significant association with a positive CACS and the adjusted mean CACS estimate was only 127 (95\% CI: 101-153) when female relatives had diabetes, despite increased numbers of diabetic female-only pedigrees (621) versus male-only pedigrees (539). The number of relatives with diabetes, type of relative, or youngest age at onset did not affect adjusted mean CACS estimates or associations with a positive CACS.

\section{DISCUSSION}

We have found numerous family history variables describing CHD in first-degree relatives that have greater CACS estimates and are significantly associated with a positive CACS compared with no family history of CHD, even after adjusting for demographic and personal cardiovascular risk factors. This includes having relatives with CHD diagnosed before or after age 60 , in men or women, or relatives from the maternal or paternal lineage. We also observed an increasing adjusted mean CACS estimate and magnitude of association with a positive CACS as the number of relatives with CHD increased and the age of onset decreased.

Previous studies have described significant associations between clinical outcomes of CHD or self-reports of CHD and increasing number of relatives with $\mathrm{CHD}^{14-19}$ and younger ages of CHD onset. ${ }^{14-17,20-22}$ An earlier study of MESA subjects has also found a significant association between a positive CACS and family history of premature CHD (defined as occurring before age 55 years in men and before age 65 years in women). ${ }^{23}$ However, this study did not assess associations between CACS and family history of later onset CHD, diabetes, or stroke. This is the first time that family history characteristics beyond premature $\mathrm{CHD}$ in a first-degree relative have been associated with the presence of coronary artery calcium.

Previous studies that have limited the definition of a positive family history to premature CHD in first-degree relatives (i.e., defined as occurring before age 55 years in men and before age 65 years in women) have found greater associations between family history and clinical CHD or subclinical atherosclerosis when siblings are affected compared to parents ${ }^{16,23,24}$ or when paternal relatives are affected compared to maternal relatives. ${ }^{25}$ We have shown the adjusted mean CACS estimate and strength of association with CACS were similar given affected parents or siblings, or maternal or paternal relatives, regardless of the age at onset. Thus, our findings suggest that familial risk assessment should not be limited to early-onset disease in siblings or paternal relatives as suggested by these previous studies.

Previous studies have found an association between family history of diabetes and cardiovascular disease as measured by endothelial dysfunction, ${ }^{26}$ common carotid artery intima-media thickness, ${ }^{27,28}$ and self-reports of CHD, ${ }^{1,29}$ and the Rancho Bernardo study found participants with family history of diabetes were more likely to have a family history of heart attack..$^{30}$ However, none of these studies assessed family history of dia- betes only, that is, in the absence of a family history of cardiovascular disease. Therefore, for the first time, we have described family history of diabetes only as a significant cardiovascular risk factor. Our findings provide further support to the "common soil" hypothesis that asserts genetic, environmental, and behavioral diabetes risk factors that are shared by family members contribute to CHD susceptibility. ${ }^{31,32}$ Interestingly, we have found that the specific diabetes family history variable of greatest significance for a positive CACS is having a male relative with diabetes. This suggests that men and women may express diabetes-related familial risk factors differently, which in turn could have differential effects on relatives at risk.

Several studies have found family history of stroke is a significant risk factor for CHD. ${ }^{1,33-36}$ We found that a family history of stroke generally had no significant associations with the adjusted mean CACS estimates or with a positive CACS. This discrepancy between reports in the literature and our results may relate to the different outcomes assessed. Previous studies have examined outcomes of clinical CHD events, such as heart attack, that are often precipitated by a thrombus superimposed on a disrupted plaque, similar to stroke. Thromboembolic factors probably do not come into play for the outcome of subclinical atherosclerosis, and this may explain why we did not find strong associations between family history of stroke and the CACS.

A major strength of this study is the large, ethnically diverse, and well-phenotyped MESA cohort. However, these MESA participants are relatively healthy, as subjects with preexisting cardiovascular disease were excluded and, therefore, this cohort is not representative of the US population. By excluding subjects with preexisting cardiovascular disease, subjects with the strongest risk factors for CACS could have been excluded, which could potentially underestimate the significance of certain familial risk factors and could potentially diminish the strength of associations we have observed between family history and CACS. Another limitation is the cross-sectional design of our study, which prohibits establishment of any temporal associations concerning family history as a risk factor.

In our study we used a measure of subclinical atherosclerosis as our outcome of interest rather than hard clinical coronary events, such as myocardial infarction, need for revascularization, or cardiovascular death. Thus, our findings are limited to this outcome. However, coronary artery calcium accumulation is part of the development of atherosclerosis, and this occurs almost exclusively in atherosclerotic arteries, particularly in advanced lesions and with older age, and is absent in the normal vessel wall. ${ }^{37-42}$ Recent studies have also shown that the CACS provides prognostic information of proven value regarding the risk of hard cardiovascular events. ${ }^{2-4,40,43-46}$ Given this evidence, several organizations, including the American Heart Association, the National Cholesterol Education Program Expert Panel, and the European Third Joint Task Force, have recommended the addition of measures of subclinical atherosclerosis as a means to improve CHD risk stratification because of the limitations of the current clinical guidelines for 
primary prevention. ${ }^{6,47,48}$ Currently in clinical practice, CACS measurement has a role as a screening or risk assessment tool that complements global risk assessment in asymptomatic patients, for the primary purpose of modifying and potentially improving selection of patients for risk-reducing therapies. ${ }^{2-4}$

Another potential limitation of our study is lack of validation of self-reports of family history. Numerous studies have estimated the accuracy of these reports. For CHD in first-degree relatives, sensitivity of self-reports range from $67 \%$ to $89 \%$, and specificity ranges form $59 \%$ to $97 \%$, with most values $>90 \%$. ${ }^{16,24,49-52}$ Sensitivity values range from $56 \%$ to $87 \%$ for family history of diabetes, and specificity ranges from $97 \%$ to $98 \%$. ${ }^{49,52}$ For family history of stroke, sensitivity ranges from $42 \%$ to $51 \%$ with specificity from $96 \%$ to $98 \% .52$ A personal history of CHD or CHD risk factors generally does not affect the accuracy of the family history report, nor does sex.49,50,52 However, older individuals are more likely to give inaccurate family history compared with younger individuals. ${ }^{49,52}$ Limited information is available regarding the influence of ethnicity/race on accuracy of family history reports. However, in the National Heart, Lung, and Blood Institute Family Heart Study, there were no significant differences in accuracy of self-reports of family history between whites and African-Americans reporting on $\mathrm{CHD}$, diabetes, and hypertension. ${ }^{49}$ Similar results were found in a study investigating the validity of cancer family history data; race or ethnicity did not influence the accuracy of reporting. ${ }^{53}$ Thus, in general, individuals are more likely to under-report and less likely to over-report disease in their relatives. Yet, despite under-reporting, positive disease associations are consistently observed, and as a result, use of family history can help to stratify disease risk in the population. However, given the lower sensitivity values and potential differences in reporting associated with age or other demographic variables, when assessing individual risk, validation of self-reports of family history is desirable, and in the clinical setting validation may be necessary if the familial risk substantially affects management decisions.

In summary, our results provide further evidence that family history is an important risk factor for CHD susceptibility. Moreover, when assessing familial risk, the family history should be considered as a categorical or continuous variable that accounts for the number of relatives with CHD and age of CHD onset, as well as family history of diabetes particularly in men. Application of rules derived from these findings could improve stratification of CHD susceptibility associated with the family history. Studies are needed to demonstrate whether adding family history could improve overall risk assessment for $\mathrm{CHD}$, which could influence CHD prevention guidelines including indications for screening to detect subclinical atherosclerosis.

\section{ACKNOWLEDGMENTS}

This work was supported by National Heart, Lung, and Blood Institute Grant 1 R21 HL081175-01A1. MESA was supported by contracts NO1-HC-95159 through N01-HC95165 and N01-HC-95169 from the National Heart, Lung, and Blood Institute. We thank the other investigators, the staff, and the participants of the MESA study for their valuable contributions.

\section{References}

1. Scheuner MT, Witworth WC, McGruder H, Yoon PW, Khoury MJ. Expanding the definition of a positive family history for early-onset coronary heart disease. Genet Med 2006;8:491-501.

2. Kondos GT, Hoff JA, Servukov A, Daviglus ML, Garside DB, Devries SS, et al. Electron-beam tomography coronary artery calcium and cardiac events: a 37month follow-up of 5,635 initially asymptomatic low- to intermediate-risk adults. Circulation 2003;107:2571-2576.

3. Greenland P, LaBree L, Azen SP, Doherty TM, Detrano RC. Coronary artery calcium score combined with Framingham score for risk prediction in asymptomatic individuals. JAMA 2004;291:210-215.

4. Arad Y, Goodman KJ, Roth M, Newstein D, Guerci AD. Coronary calcification, coronary disease risk factors, C-reactive protein, and atherosclerotic cardiovascular disease events: the St. Francis Heart Study. J Am Coll Cardiol 2005;46:158-165.

5. Expert Panel on Detection, Evaluation, and Treatment of High Blood Cholesterol in Adults. Executive summary of the third report of the National Cholesterol Education Program (NCEP). Expert Panel on Detection, Evaluation, and Treatment of High blood Cholesterol in Adults (ATPIII). JAMA 2001;285:2486-2496.

6. National Cholesterol Education Program (NCEP) Expert Panel on Detection, Evaluation, and Treatment of High Blood Cholesterol in Adults (Adult Treatment Panel III). Third Report of the National Cholesterol Education Program (NCEP) Expert Panel on Detection, Evaluation, and Treatment of High Blood Cholesterol in Adults (Adult Treatment Panel III) final report. Circulation 2002;106:3143-3421.

7. Hastie TJ, Tibshirani RJ. Generalized additive models. New York: Chapman and Hall, 1990.

8. Detrano RC, Anderson M, Nelson J, Wong ND, Carr JJ, McNitt-Gray M, et al. Coronary calcium measurements: effect of CT scanner type and calcium measure on rescan reproducibility: MESA study. Radiology 2005;236:477-484.

9. Manning WG, Morris CN, Newhouse JP, Orr LL, Duan N, Keeler EB, Leibowitz A, Marquis KH, Phelps CE. A two-part model of the demand for medical care: preliminary results from the Health Insurance Study. In: Van der Gaag J, Perlman M, editors. Health, Economics, and Health Economics. Amsterdam: North-Holland, 1981:103-123.

10. Duan N, Manning WG, Morris CM, Newhouse JP. Choosing between the sample selection model and the multi-part model. J Bus Econ Stat 1984;2:283-289.

11. Mullahy J. Much ado about two: reconsidering retransformation and the two-part model in health econometrics. J Health Econ 1998;17:247-281.

12. Graubard BI, Korn EL. Predictive margins with survey data. Biometrics 1999;55:652-659.

13. Duan N. Smearing estimate: a nonparametric retransformation method. J Am Stat Assoc 1983;78:605-610.

14. Hunt SC, Williams RR, Barlow GK. A comparison of positive family history definitions for defining risk of future disease. J Chronic Dis 1986;39:809-821.

15. Roncaglioni MC, Santoro L, D'Avanzo B, Negri E, Nobili A, Ledda A, et al. Role of family history in patients with myocardial infarction. An Italian case-control study. GISSI-EFRIM Investigators. Circulation 1992;85:2065-2072.

16. Silberberg JS, Wlodarczyk J, Fryer J, Ray CD, Hensley MJ. Correction for biases in a population-based study of family history and coronary heart disease. The Newcastle Family Heart Study I. Am J Epidemiol 1998;147:1123-1132.

17. Ciruzzi M, Schargrodsky H, Rozlosnik J, Pramparo P, Delmonte H, Rudich V, et al. Frequency of family history of acute myocardial infarction in patients with acute myocardial infarction. Argentine FRICAS Investigators. Am J Cardiol 1997;80:122127.

18. Leander K, Hallqvist J, Reuterwall C, Ahlbom A, de Faire U. Family history of coronary heart disease, a strong risk factor for myocardial infarction interacting with other cardiovascular risk factors: results for the Stockholm Heart Epidemiology Program (SHEEP). Epidemiology 2001;12:215-221.

19. Bertuzzi M, Negri E, Tavani A, LaVecchia C. Family history of ischemic heart disease and risk of acute myocardial infarction. Prev Med 2003;37:183-187.

20. Slack J, Evans KA. The increased risk of death from ischaemic heart disease in first-degree relatives of 121 men and 96 women with ischaemic heart disease. J Med Genet 1966;3:239-257.

21. Brown DW, Giles WH, Burke W, Greenlund KJ, Croft JB. Familial aggregation of early-onset myocardial infarction. Community Genet 2002;5:232-238.

22. Sesso HD, Lee I-M, Gaziano M, Rexrode KM, Glynn RJ, Buring JE. Maternal and paternal history of myocardial infarction and risk of cardiovascular disease in men and women. Circulation 2001;104:393-398.

23. Nasir K, Michos ED, Rumberger JA, Braunstein JB, Post WS, Budoff MJ, et al. Coronary artery calcification and family history of premature heart disease: sibling 
history is more strongly associated than parental history. Circulation 2004; 110:2150-2156.

24. Friedlander Y, Arbogast P, Schwartz SM, Marcovina SM, Austin MA, Rosendaal FR, et al. Family history as a risk factor for early onset myocardial infarction in young women. Atherosclerosis 2001;156:201-207.

25. Parikh NI, Hwang S-J, Larson MG, Cupples LA, Fox CS, Manders ES, et al. Parental occurrence of premature cardiovascular disease predicts increased coronary artery and abdominal aortic calcification in the Framingham Offspring and Third Generation Cohorts. Circulation 2007;116:1473-1481.

26. Goldfine AB, Beckman JA, Betensky RA, Devlin H, Hurley S, Varo N, et al. Family history of diabetes is a major determinant of endothelial function. J Am Coll Cardiol 2006;47:2456-2461.

27. Kao WH, Hsueh WC, Rainwater DL, O'Leary DH, Imumorin IG, Stern MP, et al. Family history of type 2 diabetes is associated with increased carotid artery intimalmedial thickness in Mexican Americans. Diabetes Care 2005;28:1882-1889.

28. Pannacciulli N, De Pergola G, Ciccone M, Rizzon P, Giorgino F, Giorgino R. Effect of family history of type 2 diabetes on the intima-media thickness of the common carotid artery in normal-weight, over-weight, and obese glucose-tolerant young adults. Diabetes Care 2003;26:1230-1234.

29. Park JW, Yun JE, Park T, Cho E, Jee SH, Jang Y, et al. Family history of diabetes and risk of atherosclerotic cardiovascular disease in Korean men and women. Atherosclerosis 2008;197:224-231.

30. Wingard DL, Barrett-Connor E. Family history of diabetes and cardiovascular disease risk factors and mortality among euglycemic, borderline hyperglycemic, and diabetic adults. Am J Epidemiol 1987;125:948-958.

31. Jarrett RJ. Type 2 (non-insulin-dependent) diabetes mellitus and coronary heart disease: chicken, egg or neither? Diabetologia 1984;26:99-102.

32. Stern MP. Diabetes and cardiovascular disease: the "common soil" hypothesis. Diabetes 1995;44:369-374.

33. Khaw KT, Barrett-Connor E. Family history of stroke as an independent predictor of ischemic heart disease in men and stroke in women. Am J Epidemiol 1986;123:59-66.

34. Vitullo F, Marchioli R, DiMascio R, Cavasinni L, Pasquale AD, Tognoni G. Family history and socioeconomic factors as predictors of myocardial infarction, unstable angina and stroke in an Italian population. PROGETTO 3A Investigators. Eur J Epidemiol 1996;12:177-185.

35. Wannamethee SG, Shaper AG, Ebrahim S. History of parental death from stroke or heart trouble and the risk of stroke in middle-aged men. Stroke 1996;27:1492-1498.

36. Fornage M, Lopez DS, Roseman JM, Siscovick DS, Wong ND, Boerwinkle E. Parental history of stroke and myocardial infarction predicts coronary artery calcification: The Coronary Artery Risk Development in Young Adults (CARDIA) study. Eur J Cardiovasc Prev Rehabil 2004;11:421-426.

37. Stary HC. Composition and classification of human atherosclerotic lesions. Virchows Arch A Pathol Anat Histopathol 1992;421:277-290.

38. Ross R. The pathogenesis of atherosclerosis: a perspective for the 1990s. Nature 1993;362:801-809.

39. Stary HC, Chandler AB, Dinsmore RE, Fuster V, Glagov S, Insull W Jr, et al. A definition of advanced types of atherosclerotic lesions and a histopathological classification of atherosclerosis. A report from the Committee on Vascular Lesions of the Council on Arteriosclerosis, American Heart Association. Circulation 1995;92: 1355-1374.
40. Rumberger JA, Simons DB, Fitzpatrick LA, Sheedy PF, Schwartz RS. Coronary artery calcium area by electron-beam computed tomography and coronary atherosclerotic plaque area. A histopathologic correlative study. Circulation 1995;92:21572162.

41. Bielak LF, Rumberger JA, Sheedy PF, Schwartz RS, Peyser PA. Probabilistic model for prediction of angiographically defined obstructive coronary artery disease using electron beam computed tomography calcium score strata. Circulation 2000;102: 380-385.

42. Nallamothu BK, Saint S, Rubenfire M, Fendrick AM. Electron beam computed tomography in the diagnosis of obstructive coronary artery disease. J Am Coll Cardiol 2001;37:689-690.

43. Taylor AJ, Bindeman J, Feuerstein I, Cao F, Brazaitis M, O'Malley PG. Coronary calcium independently predicts incident premature coronary heart disease over measured cardiovascular risk factors: mean 3-year outcomes in the Prospective Army Coronary Calcium (PACC) project. J Am Coll Cardiol 2005;46:807-814.

44. Vliegenthart R, Oudkerk M, Hofman A, Oei HH, van Dijck W, van Rooij FJ, et al. Coronary calcification improves cardiovascular risk prediction in the elderly. Circulation 2005;112:572-577.

45. Greenland P, Bonow RO, Brundage BH, Budoff MJ, Eisenberg MJ, Grundy SM, et al. ACCF/AHA 2007 clinical expert consensus document on coronary artery calcium scoring by computed tomography in global cardiovascular risk assessment and in evaluation of patients with chest pain: a report of the American College of Cardiology Foundation Clinical Expert Consensus Task Force (ACCF/AHA Writing Committee to Update the 2000 Expert Consensus Document on Electron Beam Computed Tomography) developed in collaboration with the Society of Atherosclerosis Imaging and Prevention and the Society of Cardiovascular Computed Tomography. J Am Coll Cardiol 2007;49:378-402.

46. Detrano R, Guerci AD, Carr JJ, Bild DE, Burke G, Folsom AR, et al. Coronary calcium as a predictor of coronary events in four racial or ethnic groups. $N$ Engl J Med 2008;358:1336-1345.

47. Smith SC Jr, Greenland P, Grundy SM. AHA Conference Proceedings. Prevention conference V: beyond secondary prevention: identifying the high-risk patient for primary prevention: executive summary. Circulation 2000;101:111-116.

48. De Backer G, Ambrosioni E, Borch-Johnsen K, Brotons C, Cifkova R, Dallongeville J, et al. European guidelines on cardiovascular disease prevention in clinical practice. Third Joint Task Force Of European and other societies on cardiovascular disease prevention in clinical practice (constituted by representatives of eight societies and by invited experts). Arch Mal Coeur Vaiss 2004;97:1019-1030.

49. Bensen JT, Liese AD, Rushing JT, Province M, Folsom AR, Rich SS, et al. Accuracy of proband reported family history: the NHLBI Family Heart Study (FHS). Genet Epidemiol 1999;17:141-150.

50. Kee F, Tiret L, Robo JY, Nicaud V, McCrum E, Evans A, et al. Reliability of reported family history of myocardial infarction. BMJ 1993;307:1528-1530.

51. Hastrup JL, Hotchkiss AP, Johnson CA. Accuracy of knowledge of family history of cardiovascular disorders. Health Psychol 1985;4:291-306.

52. Murabito JM, Nam B-H, D'Agostino RB, Lloyd-Jones DM, O'Donnell CJ, Wilson PWF. Accuracy of offspring reports of parental cardiovascular disease history: the Framingham Offspring Study. Ann Intern Med 2004;140:434-440.

53. Ziogas A, Anton-Culver H. Validation of family history data in cancer family registries. Am J Prev Med 2003;24:190-198. 